\title{
MicroRNA-218 functions as a tumor suppressor in lung cancer by targeting IL-6/ STAT3 and negatively correlates with poor prognosis
}

Yan Yang ${ }^{2}$, Lili Ding ${ }^{1}$, Qun Hu${ }^{1}$, Jia Xia ${ }^{1}$, Junjie Sun ${ }^{1}$, Xudong Wang ${ }^{4}$, Hua Xiong ${ }^{5}$, Deepak Gurbani ${ }^{6}$, Lianbo Li ${ }^{7}$, Yan Liu ${ }^{3,6}$ and Aiguo Liu ${ }^{1 *}$

\begin{abstract}
Background: Aberrant expression of microRNAs in different human cancer types has been widely reported. MiR218 acts as a tumor suppressor in diverse human cancer types impacting regulation of multiple genes in oncogenic pathways. Here, we evaluated the expression and function of miR-218 in human lung cancer and ALDH positive lung cancer cells to understand the potential mechanisms responsible for disease pathology. Also, the association between its host genes and the target genes could be useful towards the better understanding of prognosis in clinical settings.

Methods: Publicly-available data from The Cancer Genome Atlas (TCGA) was mined to compare the levels of miR218 and its host gene SLIT2/3 between lung cancer tissues and normal lung tissues. Transfection of miR-218 to investigate its function in lung cancer cells was done and in vivo effects were determined using miR-218 expressing lentiviruses. Aldefluor assay and Flow cytometry was used to quantify and enrich ALDH positive lung cancer cells. Levels of miR-218, IL-6R, JAK3 and phosphorylated STAT3 were compared in ALDH1A1 positive and ALDH1A1 negative cells. Overexpression of miR-218 in ALDH positive cells was carried to test the survival by tumorsphere culture. Finally, utilizing TCGA data we studied the association of target genes of miR-218 with the prognosis of lung cancer.

Results: We observed that the expression of miR-218 was significantly down-regulated in lung cancer tissues compared to normal lung tissues. Overexpression of miR-218 decreased cell proliferation, invasion, colony formation, and tumor sphere formation in vitro and repressed tumor growth in vivo. We further found that miR-218 negatively regulated IL-6 receptor and JAK3 gene expression by directly targeting the $3^{\prime}$-UTR of their mRNAs. In addition, the levels of both miR-218 host genes and the components of IL-6/STAT3 pathway correlated with prognosis of lung cancer patients.
\end{abstract}

Conclusions: MiR-218 acts as a tumor suppressor in lung cancer via IL-6/STAT3 signaling pathway regulation. Keywords: microRNA-218, Lung cancer, STAT3, Interleukin-6 receptor, Cancer stem cell

\footnotetext{
* Correspondence: drliuaiguo@163.com

${ }^{1}$ Department of Pediatrics of Tongji Hospital, Tongji Medical College,

Huazhong University of Science \& Technology, Wuhan 430030, People's

Republic of China

Full list of author information is available at the end of the article
} 


\section{Background}

In China, lung cancer is the most common incident cancer with high mortality rate [1].Tumor heterogeneity and poor prognosis are two major factors responsible for high mortality of lung cancer patients. In fact, among the two major lung cancer subtypes, non-small-cell lung carcinomas (NSCLC) weights more than $80 \%$ than small cell lung cancer (SCLC). The three main subgroups of NSCLC include adenocarcinoma, squamous cell carcinoma and large cell carcinoma. Adenocarcinoma is by far the more prominent type than squamous cell carcinoma [2]. Over the past several years, substantive progress has been made towards diagnosis and treatment strategies for lung cancer subtypes, but the overall 5-year survival rate of lung cancer is no more than 18\% [3]. Therefore, a better understanding of the molecular mechanisms underlying the development and progression of lung cancer is needed to improve early disease diagnosis and also provide newer therapeutic strategies for better disease control.

MicroRNAs (miRNAs) are a class of singlestranded, non-coding RNAs of 19-25 nucleotides that serve as negative regulators of gene expression by interacting with 3'untranslated regions (3'UTRs) of the target genes [4].Aberrant expression of miRNAs has been reported in different diseases including lung cancer, where they may act as oncogenes or tumor suppressors [5]. MiR-17-92 [6], miR-21 [7], and miR221/miR-222 [8] were reported to promote lung tumorigenesis, while let-7 [9], miR-126 [10], miR-16 [11], miR-340 [12], miR-145 [13], andmiR-373 [14] act as tumor suppressors.

MiR-218 is a vertebrate-specific intronic miRNA coexpressed with its host genes, tumor suppressor gene SLIT2/3. The mature form of miR-218 is generated from two separate loci, miR-218-1 and miR-218-2, which are located at the introns of SLIT2 and SLIT3, respectively [15-17]. Several lines of evidence suggests that the miR218 is depleted in some human solid cancers, such as cervical cancer [16], lung squamous cell carcinoma [17], bladder cancer [18], glioma [19], and gastric cancer [20], where tumor cell invasion and proliferation are relatively enhanced. However, the molecular mechanism of miR218 in NSCLC remains unclear.

In this present study, we attempted to determine the publicly-available data from The Cancer Genome Atlas (TCGA) for comparison of miR-218 and its host gene SLIT2/3 expression levels between lung cancer tissues and normal lung tissues. We also investigated the downstream targets of miR-218 in lung cancer cells for its underlying mechanism of action. Finally, we report the correlation between the levels of miR-218 host genes, as well as its targeted genes, and the prognosis of lung cancer disease.

\section{Methods}

\section{Cell culture, transfection and infection}

Human lung cancercell lines H1975 and A549 were purchased from the American Type Culture Collection (ATCC, Manassas, VA). Cells were cultured in RPMI 1640 medium (Gibco/Life Technologies, Grand Island, NY) supplemented with $10 \%$ fetal bovine serum (FBS) and $1 \%$ penicillin-streptomycin at $37^{\circ} \mathrm{C}$ in a humidified atmosphere with $5 \% \mathrm{CO}_{2}$. $\mathrm{H} 1975$ and $\mathrm{A} 549$ cells were transiently transfected with miR-218 mimic or miR-218 inhibitor or small interfering RNA (siRNA) (Ambion/ Life Technologies, Grand Island, NY; Sigma-Aldrich, St. Louis, MO) using Lipofectamine RNAiMAX Reagent (Life Technologies, Grand Island, NY) as per manufacturer's protocol. Co-transfection of the miRNA mimic and plasmid DNA was conducted using Lipofectamine 2000 Reagent (Life Technologies, Grand Island, NY). Lentivirus vector expressing miR-218 was purchased from Applied Biological Materials (Richmond, BC). Lentiviruses were prepared according to the manufacturer's protocol.

\section{Invasion assay}

Cell invasion assay was performed in 24-well transwell chambers(Corning, New York, NY) containing polycarbonate filters with $8 \mu \mathrm{m}$ pores coated with matrigel (Corning, New York, NY).H1975 and A549 cells were transfected with miR-218 or miR-control. Forty-eight hours after transfection, $1 \times 10^{5}$ cells suspended in serum-free medium were seeded into upper chambers. The lower chambers were filled with $600 \mu \mathrm{l}$ of RPMI 1640 containing $10 \%$ FBS as nutritional attractants. After $6 \mathrm{~h}$ of incubation, cells were fixed in 100\% pre-cooling methanol for $30 \mathrm{~min}$, and stained with crystal violet. Total cells were subjected to microscopic inspection. Five visual fields of each insert were randomly chosen under a light microscope.

\section{Colony formation assay}

Twenty-four hours after transfection or treatment with miR-218 or miR-control,H1975 and A549 cells were treated with $0.25 \%$ trypsin plus $0.5 \mathrm{mM}$ EDTA solution and re-plated in six-well plates at a density of 500 cells per well and were cultured with RPMI 1640 supplemented with $10 \%$ FBS for 10 days. At the end of the incubation period, the cells were washed twice with PBS, fixed in methanol, and dyed with crystal violet. Three independent experiments were performed.

\section{Bioinformatics analysis of miR-218 target genes}

The biological targets of miRNA targets were predicted using the algorithms TargetScan, miRDB, PicTar and PITA [21-24]. 


\section{Luciferase assay}

Double-stranded oligonucleotides corresponding to the wild-type (WT 3'-UTR) or mutant (Mut 3'-UTR) miR218 binding site in the $3^{\prime}$-UTR of IL-6R and JAK3 genes were synthesized and inserted into the PmeI and XbaI sites of the pmirGLO Vector (Promega, Madison, WI), respectively.

The sequences of the wild-type and mutated IL-6R gene 3'-UTR used were 5'-AAACTAGCGGCCGC TAGT CATGGTTCTGTCAAGCACCGCGT-3' and 5'AAACTAGCGGCCGCTAGT CGCATCGTAGATGTC CACCGCT-3', respectively (miRNA targeted and mutated bases are underlined).The sequences of the wildtype and mutated JAK3 gene $3^{\prime}$-UTR were $5^{\prime}$-AAAC TAGCGGCCGCTAGTATGGTTCCGTCAAGCACCAT GG-3' and 5'-AAACTAGCGGCCGCTAGTACATCGTA GTCAAGCACCATGG-3', respectively (miRNA targeted and mutated bases are underlined).

The wild-type or mutant luciferase reporter constructs, together with the pRL-TK Vector (Promega, Madison, WI), were co-transfected into cells with miR218 mimic or mimic-control by lipofectamine 2000 (Life Technologies, Grand Island, NY).

Forty-eight hours after transfection, firefly and renilla luciferase activity was measured by the Dual-Luciferase Reporter Assay System (Promega, Madison, WI) according to the manufacturer's protocol. Firefly luciferase activity was normalized to renilla luciferase activity. Three independent experiments were performed and the data is presented as mean $\pm \mathrm{SD}$.

\section{Real time PCR}

Twenty-four hours after miR-218 mimic transfection, total RNA was isolated from H1975 and A549 cells using the miRNeasy Mini Kit (Qiagen, Valencia, CA) according to the manufacturer's protocol. RNA concentrations were determined using the NanoDrop. Briefly, $500 \mathrm{ng}$ of total RNA from each sample was subjected to reverse transcription using a High Capacity cDNA Reverse Transcription Kit (Applied Biosystems, Foster City, CA). Expression of the IL-6R or JAK3 gene was detected using $\mathrm{RT}^{2} \mathrm{qPCR}$ Primer Assays and $\mathrm{RT}^{2}$ SYBR Green Mastermixes (Qiagen, Valencia, CA) in an ABI 7900HT Sequence Detection System (Applied Biosystems, Foster City, CA). Relative gene expression was normalized to the expression of GAPDH and was calculated using the $2^{(-\Delta \Delta \mathrm{CT})}$ method.

\section{Western blotting}

Cells were lysed using the RIPA Lysis and Extraction Buffer (Life Technologies, Grand Island, NY) supplemented with protease inhibitors (Roche, Indianapolis, IN). The total protein was quantified with a Pierce BCA Protein Assay Kit (Pierce Biotechnology, Rockford, IL) according to the manufacturer's protocol. Protein samples were separated by sodium dodecyl sulfatepolyacrylamide gel electrophoresis (SDS-PAGE) and transferred to a polyvinylidene fluoride (PVDF) membrane. After blocking in phosphate-buffered saline/ Tween-20 containing 5\% non-fat milk at room temperature for $1 \mathrm{~h}$, the membrane was incubated with primary antibody (Cell Signaling Technology, Danvers, MA) at $4{ }^{\circ} \mathrm{C}$ overnight using human IL-6R antibody (1:2000, \#12786), JAK3 antibody (1:2000, \#8863), STAT3 antibody (1:2000, \#4904), pSTAT3 antibody (1:1000, \#9145), EGFR antibody (1:2000, \#4267). Then, the membrane was incubated with goat anti-rabbit IgG secondary antibody conjugated with horseradish peroxidase (1:5000; Santa Cruz Biotechnology, Dallas, Texas). GAPDH (\#2118) was used as a loading control. Proteins were visualized with LumiGLOchemiluminescent substrate (Cell Signaling Technology, Danvers, MA).

\section{Immunofluorescence}

Forty-eight hours after miR-218 mimic transfection, cells were fixed with 50\% methanol and 50\% acetone at $4{ }^{\circ} \mathrm{C}$ for $30 \mathrm{~min}$ and blocked with $1 \%$ BSA for $1 \mathrm{~h}$. After incubation with pSTAT3 or Ki67 primary antibody(Cell Signaling Technology, Danvers, MA), the cells were incubated with FITC or Alexa Fluor(R) 555-coupled secondary antibody(Cell Signaling Technology, Danvers, MA) for $1 \mathrm{~h}$ and then stained with the nuclear marker DAPI(Cell Signaling Technology, Danvers, MA) for $10 \mathrm{~min}$. Fluorescence was observed with microscope.

\section{Aldefluor assay and FACS sorting}

Aldefluor assay and Flow cytometry was used to quantify and enrich ALDH positive cancer cells with stem cell characterics since aldehyde dehydrogenase (ALDH) is a cancer stem cell associated marker in lung cancer [25, 26].The ALDH activity of H1975 and A549 was determined by using the Aldefluor assay kit (Stem Cell Technologies, Durham, NC). In brief, cells were dissociated into single cells by trypsin/EDTA digestion. Then, the single-cell suspension was washed twice in PBS without $\mathrm{Ca}^{2+} / \mathrm{Mg}^{2+}$ and suspended in $1 \mathrm{ml}$ Aldefluor assay buffer containing $5 \mu \mathrm{l}$ ALDH substrate $\left(1 \mathrm{ml} /\right.$ per $1 \times 10^{6}$ cells $)$ and incubated for 30-40 min at $37{ }^{\circ} \mathrm{C}$ in the dark. As a negative control, cells were treated with $5 \mu \mathrm{l}$ of diethylaminobenzaldehyde (DEAB; $50 \mathrm{mmol} / \mathrm{l}$ ), a specific ALDH inhibitor. For FACS sorting, cells were re-suspended in PBS buffer at $1 \times 10^{7}$ cells per $\mathrm{ml}$ and run on an Aria cell sorter (BD Biosciences). The sorting gates were established, by negative control cells which were treated with the ALDH inhibitor DEAB. 


\section{Tumorsphere culture}

After FACS sorting, the ALDH ${ }^{+} \mathrm{H} 1975$ cells and ALDH

+ A549 cells were placed in ultralow attachment 6-well plates (Corning, NY)at a density of 2000 cells per well in serum-free stem cell medium (MEBM). Twenty-four hours after seeding, the ALDH1A1 positive cells were infected with lentivirus expressing miR-218 or miRcontrol. Every three to four days, half of the medium was replaced. After 10-14 days, spheroid formation was checked and representative images were taken.

\section{Mouse xenograft tumor model}

All the animal studies were conducted according to protocols approved by the Ethical Committee of Hua Zhong University of Science and Technology, People's Republic of China. And all the animals were kept according to the Animal Care Guidelines and housed in $12 \mathrm{~h}$ light/dark conditions with free access to food and water. Human lung cells A549 were infected with lentivirus expressing miR-218 or miR-control. Forty-eight hours after infection, cells were harvested and were counted by Handheld Automated Cell Counter Scepter ${ }^{\mathrm{TM}}$ (Millipore, Bilerica, MA). $5 \times 10^{6}$ cells were subcutaneous injected into 6 weeks old nude mice (SJA Lab Animal, Hunan, China). Tumor growth was monitored once a week by caliper measurements $(\mathrm{LxWxD})$. At fifth week after injection, mice were sacrificed and tumors were collected for analysis.

\section{Statistical analysis}

Differences between experimental groups and controls were assessed by Student's t-test. Two-tailed tests were used, and a $P$-value of 0.05 or less was considered statistically significant. Data is shown as mean \pm SD of three independent experiments.

\section{Results}

Expression levels of miR-218 and its host gene SLIT2/3 in normal and lung tumor tissues

The levels of miR-218 were found to be significantly lower in lung adenocarcinoma tissues and were compared to normal lung tissues (fold change of cancer vs. normal $=0.53, p=3.2 \times 10^{-8}$ ) (Fig. 1a) using the existing data from TCGA and following the relevant algorithms (http://starbase.sysu.edu.cn) [27, 28]. To further investigate lung tumorigenesis, we also analyzed the expression of SLIT2 and SLIT3 since miR-218 co-expresses with these host tumor suppressor genes. The data revealed that the levels of SLIT2 and SLIT3 were also significantly lower with fold change of SLIT2 to be 0.14 $\left(p=1.3 \times 10^{-15}\right)$ in lung adenocarcinoma tissues when compared to normal lung tissues (Fig. 1b). Similarly, the fold change of SLIT3 in cancer vs. normal tissues was $0.16\left(p=8.9 \times 10^{-16}\right)$ (Fig. 1c).
Additionally, we also compared the levels of miR218, SLIT2 and SLIT3 in lung squamous cell carcinoma subtype and normal tissues. Similar low expression levels of miR-218, SLIT2and SLIT3 were obtained in lung cancer tissues when compared to normal lung tissues (the fold change of miR-218 in cancer vs. normal $=0.29, p=4.4 \times 10^{-16}$, Fig. $1 \mathrm{~d}$; the fold change of SLIT2 in cancer vs. normal $=0.10$, $p=1.8 \times 10^{-15}$, Fig. 1e; the fold change of SLIT3 in cancer vs. normal $=0.08, p=8.9 \times 10^{-16}$, Fig. 1f) . These data suggest that both miR-218 and the related host genes were downregulated in both of lung cancer NSCLC subtypes.

Overexpression of miR-218 affects lung cancer cell proliferation and invasiveness

To study whether re-expression of miR-218 would affect cell behavior, we transfected miR-218 or antimiR-218 into A549 and H1975 cells and then performed cell proliferation, trans-well invasion, and colony formation assays. The results of cell proliferation showed that re-expression of miR-218 reduced cell proliferation (Fig. 2a), whereas overexpression of anti-miR-218 promoted cell proliferation (Fig. 2b). Our data also demonstrated that overexpression of miR-218 decreased cell invasion (Fig. 2c) and colony formation ability (Fig. 2d).

\section{MiR-218 negatively regulates IL-6/JAK/STAT3 pathway}

To further gain insights by which miR-218 could potentially regulate the cell growth and differentiation of lung cancer cells, we performed predictions using programs TargetScan, miRDB, PicTar and PITA. It was predicted that miR-218 could target components of IL-6/JAK/STAT3 pathway. TargetScan showed that both IL-6R and JAK3 had a score of 94, respectively (Fig. 3a). Therefore, it could be asserted that miR218 could portray its inhibitory effects by at least partially modifying IL-6/JAK/STAT3 signaling mediator molecules. To determine whether the IL-6R and JAK3 mRNA expression is regulated by miR-218 through direct binding to their 3 '-UTR regions, we used a dual-luciferase reporter system containing either the wild-type or the mutated $3^{\prime}$-UTR of IL-6R and JAK3. The relative luciferase activity decreased in H1795 cells transfected with luciferase reporter pmirGLO-3' UTR-wild-type and miR-218 compared with the negative control miR-cont. This suppressive effect was abolished by the mutations in the miR218 targeted 3' UTR regions (Fig. 3b). To further gain evidence, we examined the mRNA and protein expression of IL-6/JAK/STAT3 pathway after overexpressing miR-218 by qRT-PCR and western blotting. Overexpression of miR-218 significantly reduced the 


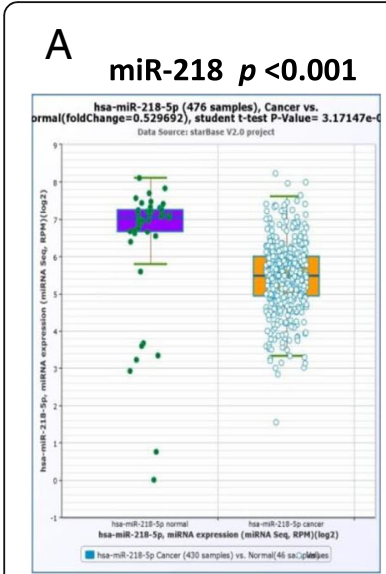

1

D

$\operatorname{miR}-218 p<0.001$

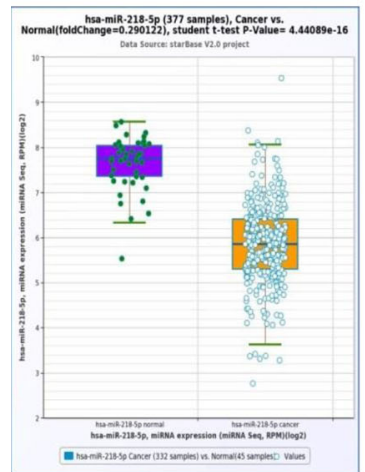

1
B

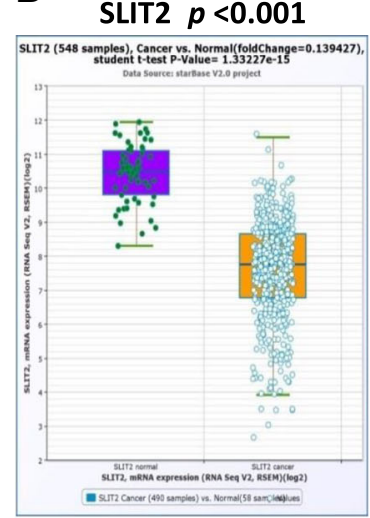

1

E

SLIT2 $p<0.001$

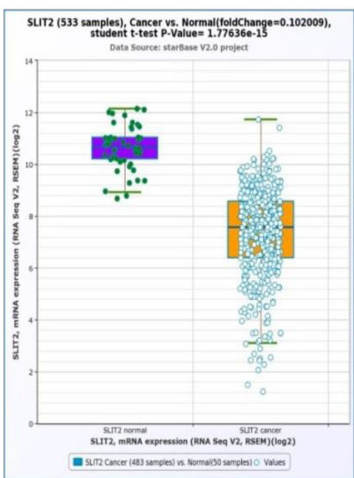

1
2

2

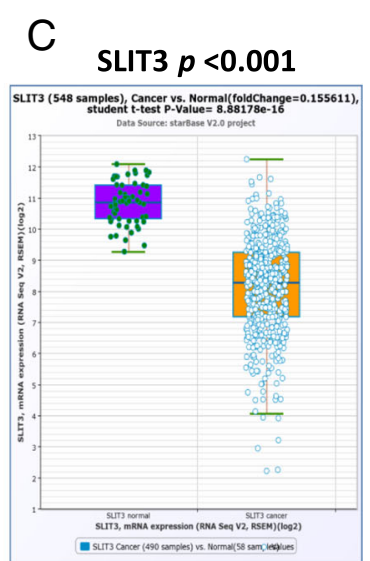

1

2

\section{$\mathrm{F}$}

SLIT3 $p<0.001$

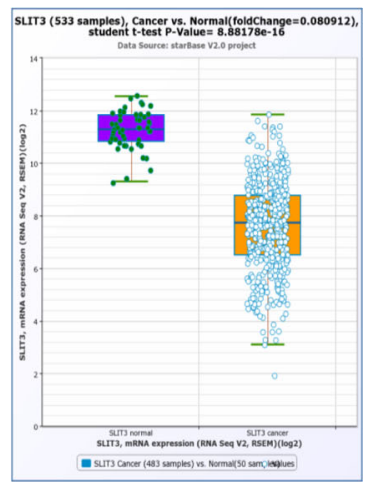

1: Normal

2: Lung squamous cell carcinoma

Fig. 1 MiR-218 correlates with tumorigenesis in lung cancer. a The expression levels of miR-218 in 430 lung adenocarcinoma tumor tissues and 46 normal tissues were obtained from TCGA. b-c The expression levels of SLIT2 and SLIT3 in 490 lung adenocarcinoma tumor tissues and 58 normal tissues were obtained from TCGA. (d) The expression levels of miR-218 in 332 lung squamous cell carcinoma tumor tissues and 45 normal tissues were obtained from TCGA. e-f The expression levels of SLIT2 and SLIT3 in 483 lung squamous cell carcinoma tumor tissues and 50 normal tissues were obtained from TCGA

expression of JAK3, IL-6R and phosphorylated STAT3 (Fig. 3c, d). Furthermore, immunofluorescence staining revealed that overexpression of miR218 decreased phosphorylation of STAT3 (Fig. 3e). To validate these findings we examined whether activating STAT3 signal with IL-6 treatment can rescue cells upon miR-218 transfection, we treated miR-218 transfected cells with IL-6. Our data showed that IL6 treatment partially rescued cells transfected by miR-218 (Fig. 3f). Taken together, these data suggest that miR-218 modulated lung cancer cell phenotype through negatively regulating the STAT3 signaling pathway. Additionally, we also investigated the effect of STAT3 pathway inhibition in lung cancer cells to understand the importance of IL-6/JAK/STAT3 pathway. We decreased IL-6R and JAK3 with siRNA or decreased phosphorylated STAT3 with a STAT3 inhibitor, LLL12 [29, 30].Decreasing either IL-6R or JAK3 reduced the levels of phosphorylated STAT3 (Fig. 4a). Cell based assays demonstrated that treating cells with siR-IL-6R, siR-JAK3 or LLL12 inhibited cell proliferation (Fig. 4b-d) and colony formation (Fig. 4e-g). These results confirmed the establishment of IL-6/STAT3 signaling in currently studied lung cancer cells. In addition to IL-6R and JAK3, miR-218 was also reported to negatively regulate EGFR, leading to reduced levels of pSTAT3 [31]. We confirmed the previous results (Additional file 1: Figure S1A) and found downregulation of EGFR decreased the levels of pSTAT3 more significantly in EGFR mutated cells (H1975) than in EGFR wild type cells (A549) (Additional file 1: Figure S1B). Similarly, 


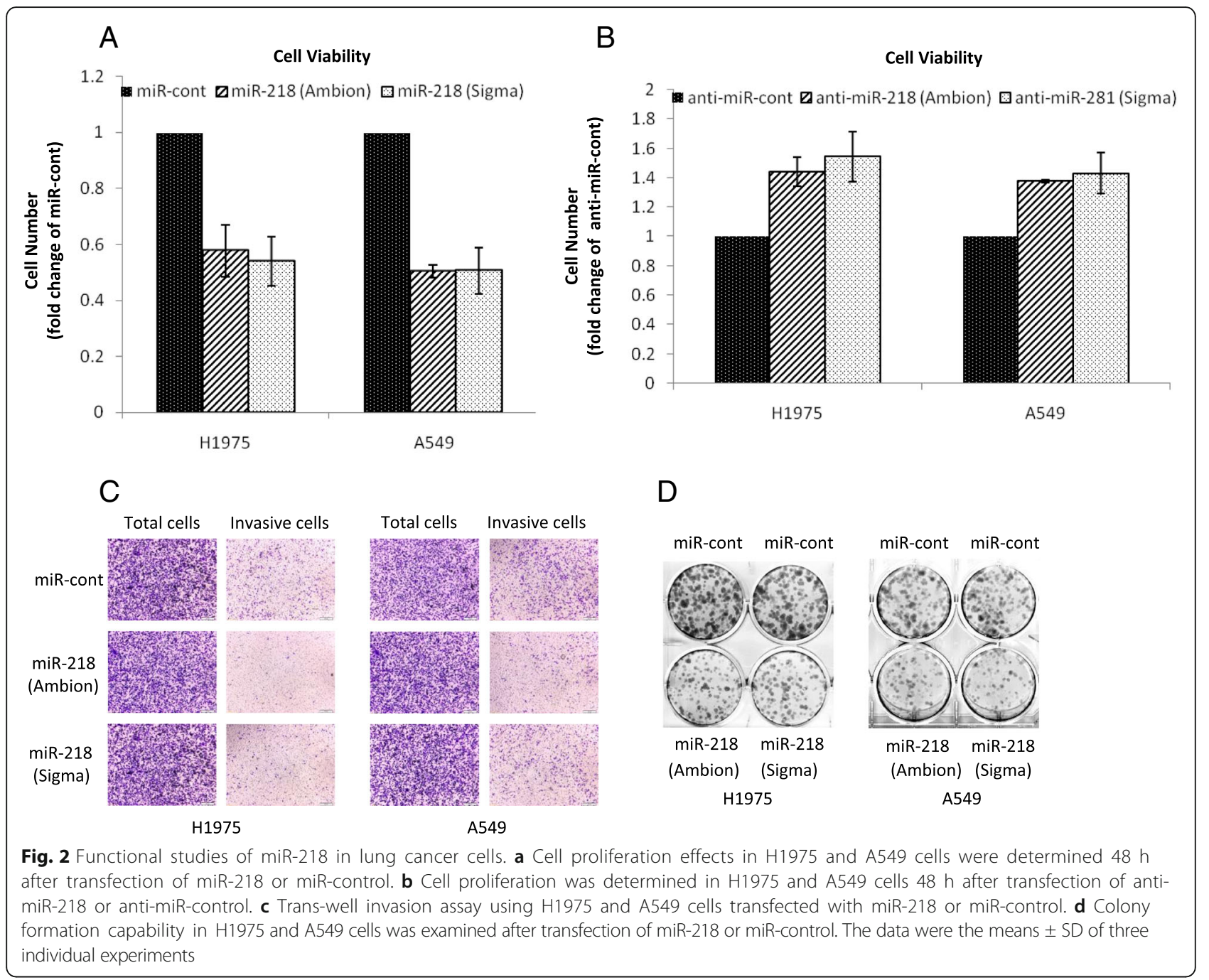

downregulating of EGFR reduced cell viability more significantly in EGFR mutated cells (H1975) than in EGFR wild type cells (A549) (Additional file 1: Figure S1C). Overall, our data indicated that miR-218 negatively regulated STAT3 signaling through IL-6R and JAK3 in EGFR wild type cells and through IL6R, JAK3, and EGFR in EGFR mutated cells.

MiR-218 is downregulated in ALDH positive lung cancer cells ALDH positive lung cancer cells have shown some of the characterics of cancer stem cells, such as drug resistance [26]. To explore whether miR-218 would be dysregulated in these small populations of cells marked by ALDH1A1, we isolated ALDH1A1 positive cells from both H1975 and A549 cells by flow cytometry. We compared the miR-218 levels in ALDH1A1 positive and ALDH1A1 negative cells by Real Time PCR and found that ALDH1A1 positive cells had lower levels of miR-218 (Fig. 5a). We further observed that ALDH1A1 positive cells had higher levels of IL-6R, JAK3 and phosphorylated STAT3 (Fig. 5b), suggesting that the lower levels of miR-218 may be responsible for the upregulation of STAT3 signaling in ALDH positive lung cancer cells. We overexpressed miR-218 in ALDH1A1 positive cells by lentivirus expressing miR-218 (Fig. 5c) and found that the levels of IL-6R, JAK3 and phosphorylated STAT3 were reduced (Fig. 5d). Additionally, overexpression of miR218 inhibited ALDH1A1 positive to survive in anchorage-independent conditions and their ability to form tumor-spheres (Fig. 5e).

Overexpression of miR-218 reduces tumor growth in vivo by targeting STAT3 signaling

To investigate whether overexpression of miR-218 would reduce tumor growth in vivo, we infected 


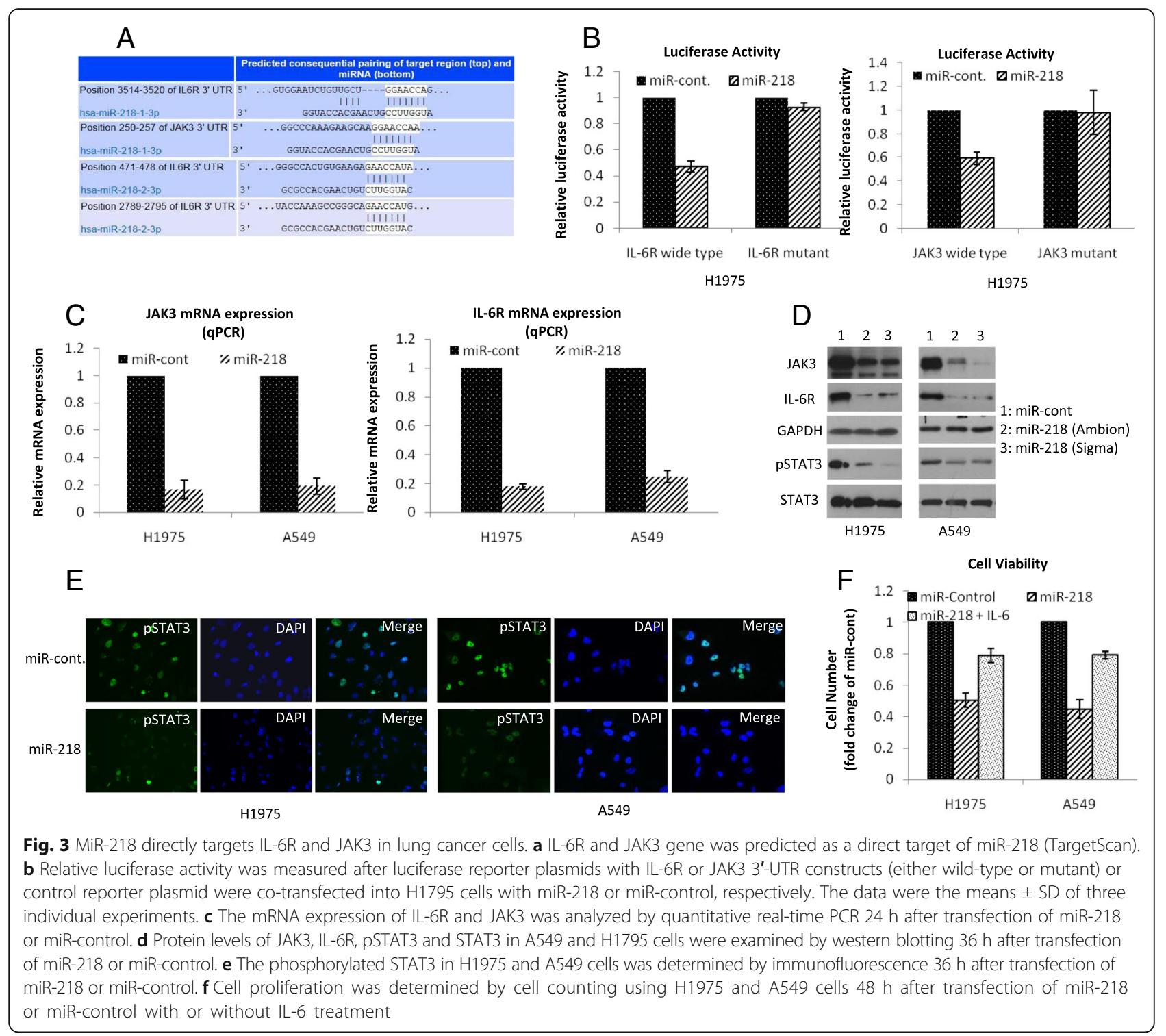

A549 cells with lentivirus expressing miR-218. Cells infected with miR-218 expressing virus showed reduced cell proliferation compared to cells infected with control virus (Fig. 6a). Further evidence was acquired by injecting these infected cells subcutaneously into nude mice. Compared to the control group, the mean volume of the tumors in the miR218 overexpressing group was significantly smaller (Fig. 6b). Subsequently, we assessed STAT3 signaling and proliferation via staining for phosphorylated STAT3 (Fig. 6c) and Ki-67 (Fig. 6d). Compared with the tumors from the control group, tumor tissues with miR-218 overexpression showed lower levels of phosphorylated STAT3(Fig. 6c) and Ki67 (Fig. 6d), suggesting that overexpressing miR-218 inhibited the STAT3 signaling and reduced tumor growth in vivo.

MiR-218 host genes and the molecules of IL-6/STAT3 signaling pathway correlate with prognosis of lung cancer patients

In order to correlate our findings with lung cancer prognosis in clinical settings, we analyzed the data from 1405 lung cancer patients and the gene expression of miR-218 host genes (SLIT2/SLIT3), IL-6, IL-6R, JAK3 and STAT3 in their tumor tissues [32]. Median levels of gene expression were used for all six genes as cutoff points. Both miR-218 and host genes, SLIT2 and SLIT3 were negatively correlated with poor prognosis (Fig. 7a, b), whereas $I L-6, I L-6 R, J A K 3$ and STAT3 were positively 


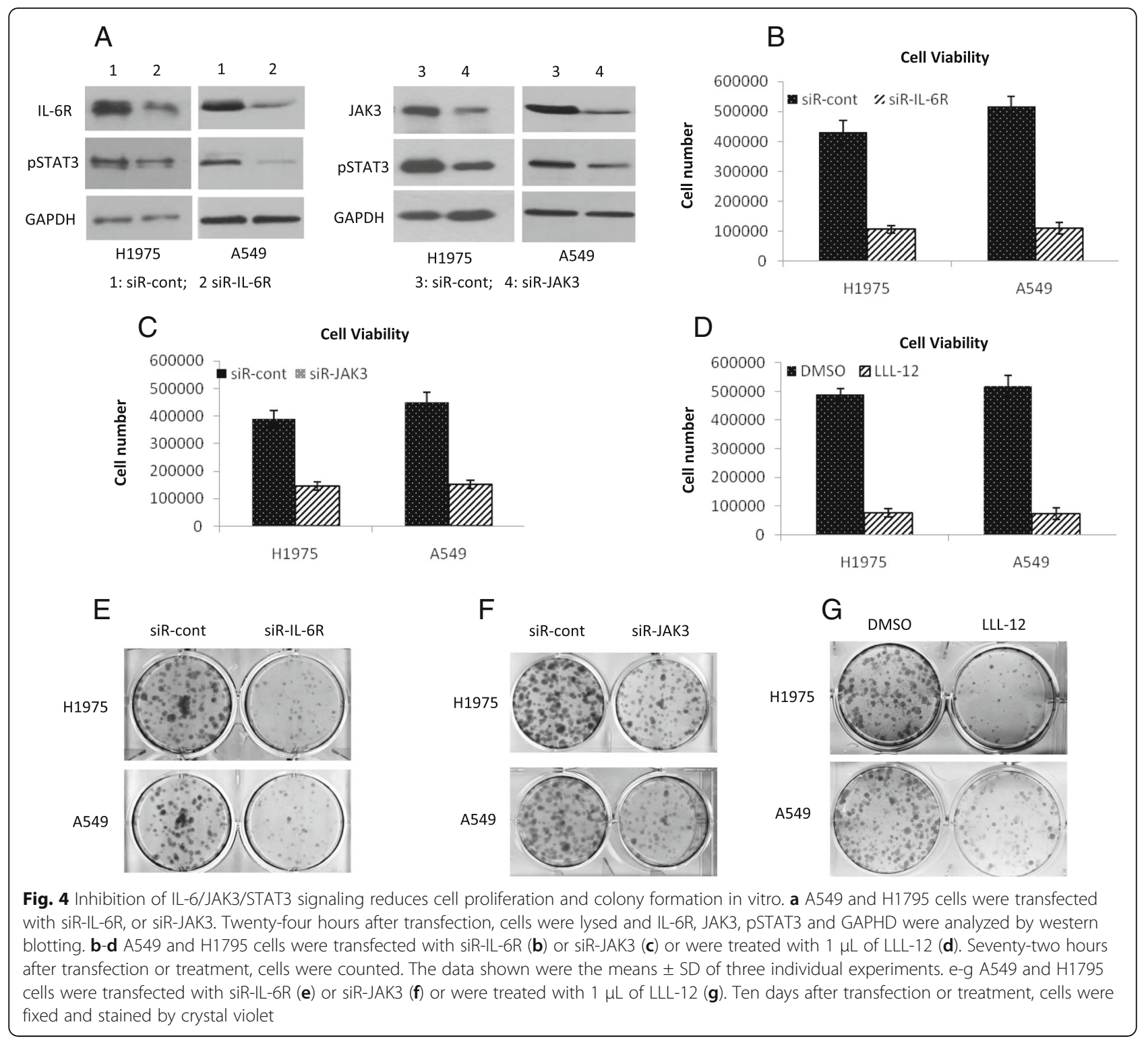

correlated with poor prognosis (Fig. 7c-f). We also analyzed the correlation between levels of IL6-R and miR218, as well as the correlation between levels of JAK3 and miR-218 in patients with lung adenocarcinima TCGA cohort, which showed significant negative correlation (Additional file 1: Figure S2).

\section{Discussion}

In normal cell physiology, miRNAs regulate the precision of gene expression to modulate cell signaling pathways for regular growth and differentiation [33]. However, cancer cells display dysregulation of miRNA expression through different mechanisms, including abnormal transcription, epigenetic changes, amplification or deletion of miRNA host genes [34]. The advances in this field have brought new diagnostic and therapeutic opportunities for patients affected with different types of cancer. Clinical trials using miRNAs started in 2013 and studies have been conducted in breast cancer, liver cancer, glioma, and non-small-cell lung cancer towards identifying miRNAs as biomarkers. In such patients new opportunities towards molecular diagnostics and therapies could emerge when investigating responses of miRNAs and underlying molecular mechanisms behind cancer progression [35]. In this study, we evaluated the diverse roles of miR-218 in human lung cancer, investigated the molecular mechanisms, and determined whether it could serve as a biomarker of prognosis providing newer insights towards therapeutics. 


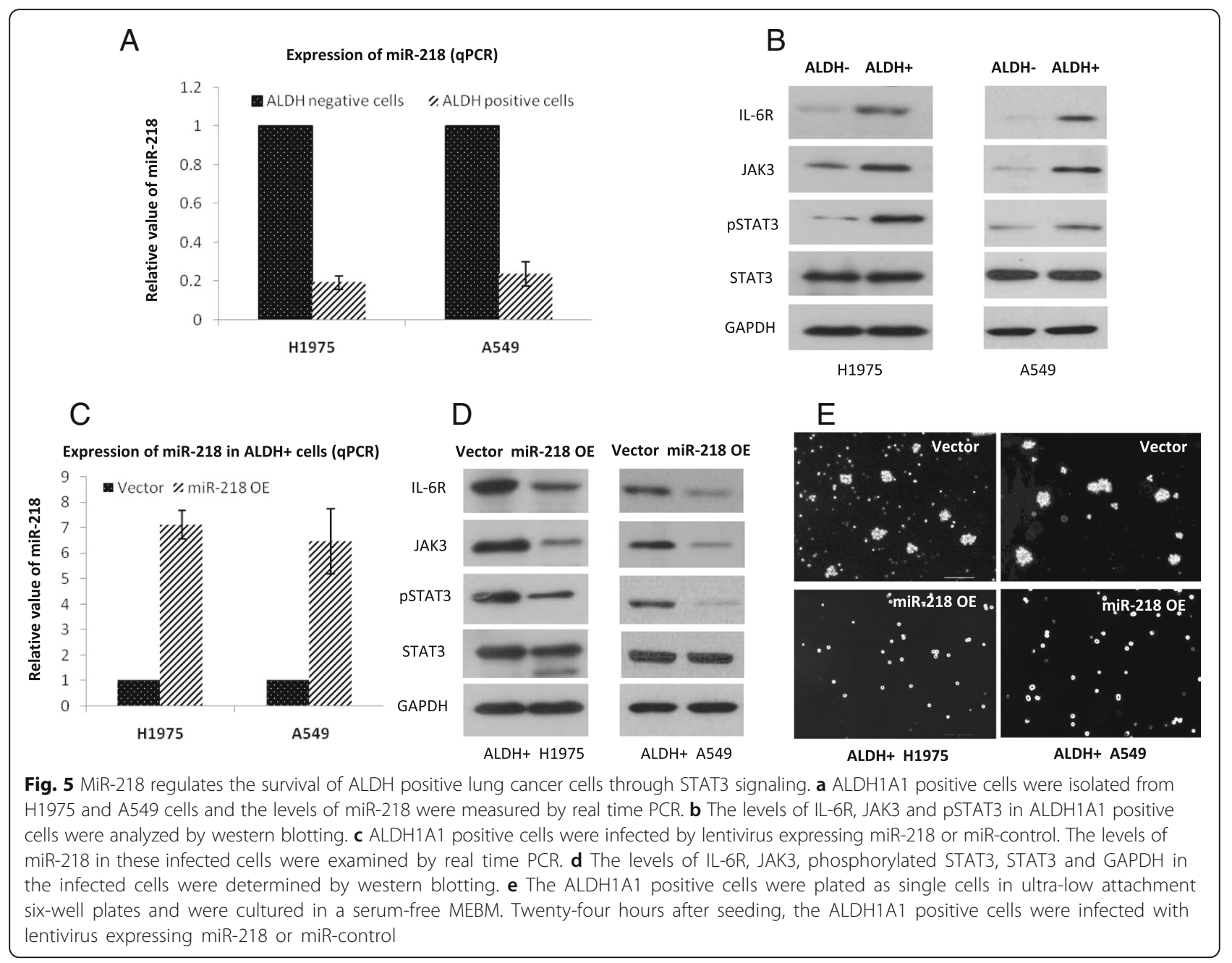

Lung cancer is the most leading cancer death in men all over the world, especially in China $[1,36]$. Despite recent advances in the diagnostics and treatment, the prognosis of patients with lung cancer remains poor. For example, almost all NSCLC patients while initially responding to EGFR inhibitor treatment eventually develop disease resistance [37]. Studies involved in evaluating the role of miRNAs showed dysregulation of miRNA expression in NSCLC and postulated its important role toward malignancy [38]. These miRNAs serve as either oncogenes or tumor suppressors. It is seen that the expression of miR-17, miR-18a, miR-19, miR-20a, miR-21, miR-31, miR-92a, and miR-224 is upregulated in lung cancer cells and inhibition of their expression can reduce cell growth and invasion capacities [7, 39-41]. Others, such as let-7, miR-34, and miR-126 are downregulated in human lung cancer and their overexpression is implicated in reducing cellular growth and invasion capacities [42-44]. A previous study highlighted that the levels of miR-218 is downregulated in the tissues of lung squamous cell carcinoma, in association with cigarette smoking [17]. It should be noted that several factors other than cigarette smoking are responsible factors towards development of NSCLC. Our study using lung cancer samples included in TCGA database reaffirmed the results of the previous study in lung squamous cell carcinoma and also found that the levels of miR-218 and its host genes SLIT2/3 are downregulated in the tissues of lung adenocarcinoma. Overexpression of miR-218 in lung cancer cells reduced cell proliferation and invasiveness, suggesting miR-218 is acting as tumor suppressor consistent with its function in other reported human cancer types [16, 18-20].

MiR-218 has been reported to target EGFR [31], an important kinase implicated in signaling in lung cancer cells. We found that miR-218 also targeted two molecules of IL-6/JAK/STAT3 signaling pathway, which is constitutively activated in NSCLC [45]. Abnormalities in the IL-6/JAK/STAT3 pathway are also due to expression of a variety of oncogenes, such as Myc and VEGF, 


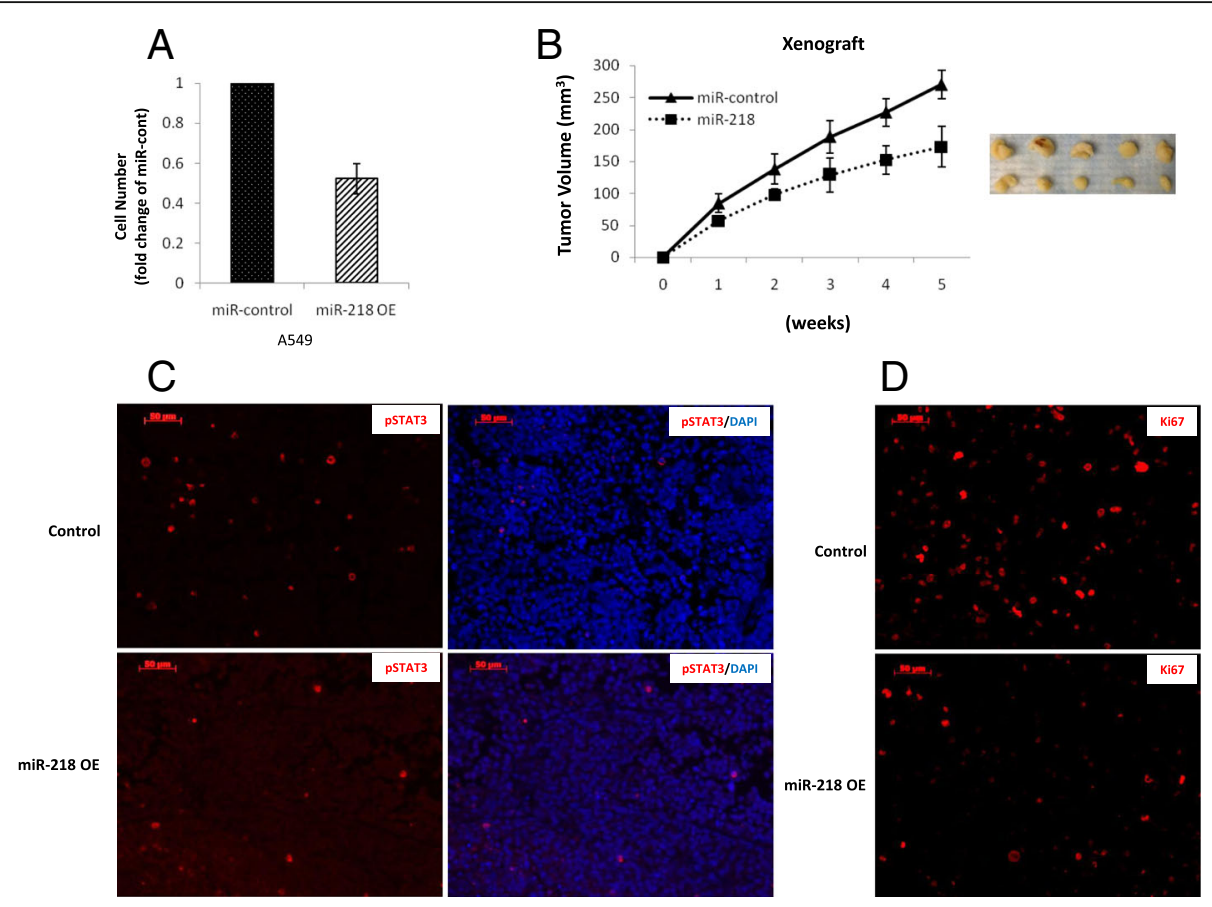

Fig. 6 Overexpression of miR-218 represses tumor growth in vivo. a A549 cells were infected with lentivirus expressing miR-218 or miR-control. Forty-eight hours after infection, cell proliferation was determined. b $5 \times 10^{6}$ A549 cells infected with lentivirus expressing miR-218 or miR-control were injected subcutaneously into two groups of nude mice (five mice per group). Tumor volumes were measured once per week. $\mathbf{c}$ and $\mathbf{d}$ Tissue levels of phosphorylated STAT3 and Ki67 in an A549 xenograft were visualized by immunofluorescence co-staining with DAPI

reportedly increased in lung cancer and other cancer types [46, 47]. Persistent activation of STAT3 can be caused by either the reduced levels of negative regulators, such as PIAS, or the increased levels of kinases or receptors [45]. Our data revealed a novel mechanism, by which STAT3 is persistently activated in lung cancer. MiR-218 was downregulated in NSCLC, which then caused constitutive activation of STAT3 signaling. Overexpressing miR-218 in lung cancer reduced STAT3 signaling when tested both in vitro and in vivo. In addition, in EGFR mutated lung cancer cells, miR-218 can also negatively regulate pSTAT3 signaling through targeting EGFR [31].

More importantly, we found that ALDH positive lung cancer cells, marked by ALDH1A1, had lower levels of miR-218 and higher levels of STAT3 signaling than in ALDH negative cells. This small population may be responsible for tumor maintenance. These cells are hypothesized to persist in tumors as a distinct population and cause relapse and metastasis by giving rise to new tumors [48]. Therefore, development of specific therapies targeting these cells holds hope for improvement of survival and quality of life of cancer patients, especially for patients with metastatic disease.The dysregulation of miRNAs have been implicated in regulating cancer stem cells or drug resistant cells [49].Therefore, a better understanding of miRNA expression in cancer stem cells or drug resistant cells and the molecular mechanisms may help identify new therapeutic targets. Our findings that miR-218 was downregulated in ALDH positive lung cells aid to clarify the mechanism of upregulation of STAT3 signaling in these cells. It has been shown that targeting JAK/STAT3 with inhibitors results in downregulating self-renewal capability of lung cancer stem cells and displays reduced resistance to multiple cancer drugs [50]. Our data suggest that overexpressing miR-218 in ALDH positive lung cancer cells could also exhibit similar responses in reducing self-renewal capability of these cells.

In a previous study, miR-218 was reported to positively regulate STAT3 in the spinal cord and microglia through targeting SOCS3 [51]. However, SOCS3, a tumor suppressor, is silenced in NSCLC $[52,53]$. Therefore, miR-218 is not able to positively regulate STAT3 in NSCLC, whose SOCS3 is silenced.

In summary, miR-218 is capable to inhibit lung cancer cell proliferation and invasion, at least partially through repressing IL-6R and JAK3 genes expression. The newlyidentified miR-218-mediated IL-6R and JAK3 genes silencing may facilitate a better understanding of the molecular mechanisms of lung cancer progression and present a new strategy to treat patients with lung cancer. 


\section{A}

SLT2 expression associated with recurrence-free survival

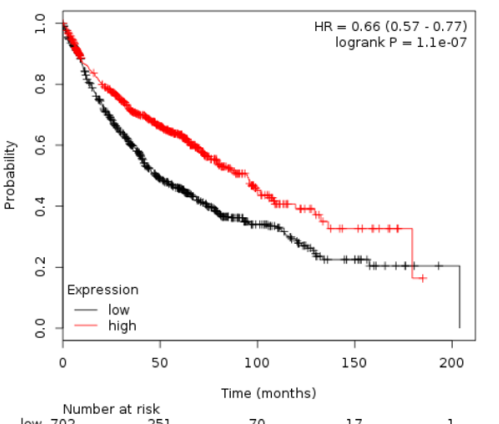

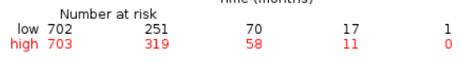

D

IL-6R expression associated with recurrence-free survival

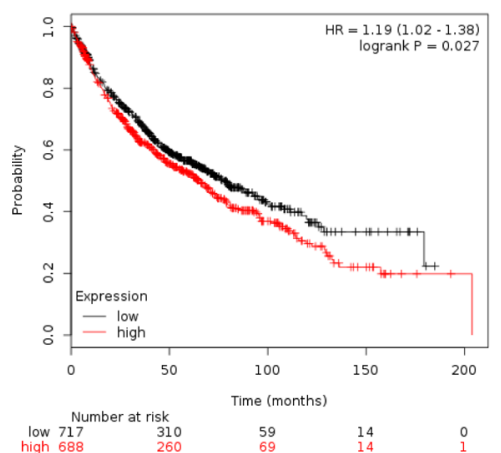

B

SLIT3 expression associated with recurrence-free survival

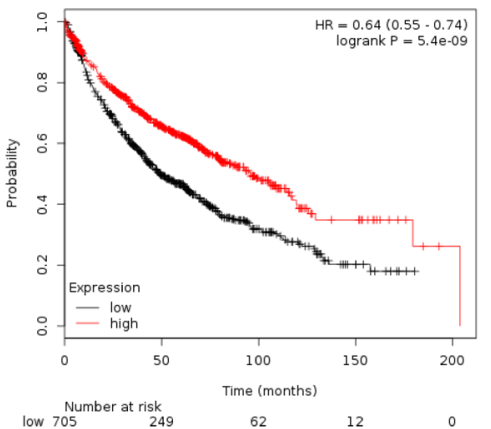

$E$

JAK3 expression associated with recurrence-free survival

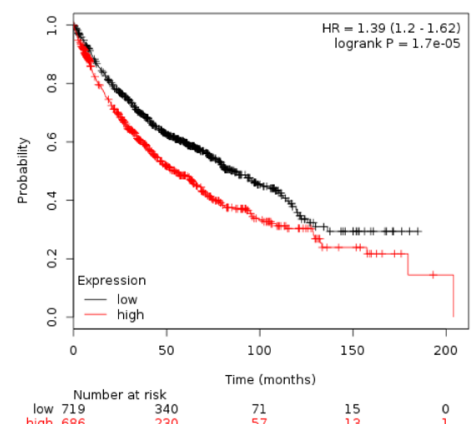

C

IL-6 expression associated with recurrence-free survival

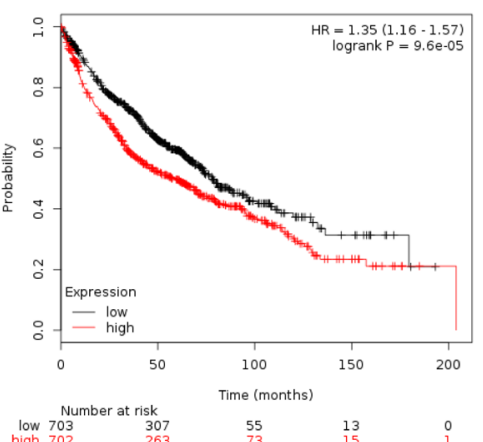

$\mathrm{F}$

Fig. 7 The components of the IL-6/JAK/STAT3 pathway and miR-218 host genes correlate with prognosis in lung cancer patients. a Kaplan-Meier curves for recurrence free survival were created using the Kaplan-Meier Plotter (www.kmplot.com) with lung cancer patients classified according to high and low SLIT2 (a), SLIT3 (b), IL-6 (c), IL-6R (d), JAK3 (e) and STAT3 (f) gene expression. Hazard ratio (with 95\% confidence interval) and log-rank $p$ values were calculated

\section{Conclusions}

Overall, our findings of the anti-proliferative effects of miR-218 in lung adenocarcinima cells, through the direct targeting of IL-6R and JAK3, further corroborate a tumor suppressive role for miR-218 in NSCLC. Especially considering the significant decrease of miR-218 in ALDH positive cells with cancer stem cell characterics, further investigation of IL-6/STAT3 as a potentially therapeutic target in NSCLC is warranted.

\section{Additional file}

Additional file 1: Figure S1. (A) A549 and $\mathrm{H} 1975$ cells were transfected with miR-cont or miR-218. Protein levels of EGFR were examined by western blotting $36 \mathrm{~h}$ after transfection. (B) A549 and H1975 cells were transfected with siR-cont or siR-EGFR. Protein levels of EGFR and PSTAT3 were examined by western blotting $36 \mathrm{~h}$ after transfection. (C) Cell proliferation in H1975 and A549 cells was determined $48 \mathrm{~h}$ after transfection of siR-EGFR or siRcontrol. Figure S2. Correlation of IL-6R/JAK3 and miR-218 in patients with lung adenocarcinoma in TCGA cohort. (PPT $4631 \mathrm{~kb})$

\section{Abbreviations}

ALDH: Aldehyde dehydrogenase; IL-6R: Interleukin-6 receptor; JAK STAT3: Janus kinase /signal transducer and activator of transcription 3; MiR218: microRNA-218; NSCLC: Non-small-cell lung carcinomas; TCGA: The Cancer Genome Atlas

\section{Acknowledgements}

The authors would like to thank Ai Zhang, Yaqin Wang, Songmi Wang, and Ping Chen for technical and FACS analysis support.

\section{Funding}

This work was supported by a General Program from the National Natural Science Foundation of China (No. 81472706).

\section{Availability of data and materials}

The datasets during and/or analyzed during the current study are available from the corresponding author on reasonable request.

\section{Authors' contributions}

YY performed experiments, wrote the manuscript and contributed to the design of the study. LD performed cell culture and gene knockdown experiments. QH critically reviewed the manuscript and assisted in study design. JX performed animal experiments.JS performed plasmid construction. $\mathrm{XW}$ critically reviewed the manuscript and assisted in study design. HX performed bioinformatics analyses and critically reviewed the manuscript. DG critically reviewed the manuscript. LL critically reviewed the manuscript and 
contributed to study design. YL performed experiments, designed the study and wrote the manuscript. AL designed the study, performed experiments and wrote the manuscript. All authors read and approved the final manuscript.

\section{Ethics approval and consent to participate}

The animal experiment was approved by the local ethical committee of Huazhong University of Science and Technology. Anonymised patient data from TGCA studies were available from the Genomic Data Commons Data Portal.

\section{Consent for publication}

Not applicable.

\section{Competing interests}

The authors declare that they have no competing interests.

\section{Publisher's Note}

Springer Nature remains neutral with regard to jurisdictional claims in published maps and institutional affiliations.

\begin{abstract}
Author details
'Department of Pediatrics of Tongji Hospital, Tongji Medical College, Huazhong University of Science \& Technology, Wuhan 430030, People's Republic of China. ${ }^{2}$ Experimental Medicine Center of Tongji Hospital, Tongji Medical College, Huazhong University of Science \& Technology, Wuhan 430030, China. ${ }^{3}$ Department of Medicine, Division of Epidemiology, Vanderbilt University, Nashville, TN 37232, USA. ${ }^{4}$ Department of Biochemistry, UT Southwestern Medical Center, Dallas, TX 75390, USA. ${ }^{5}$ Department of Oncology of Tongji Hospital, Tongji Medical College, Huazhong University of Science \& Technology, Wuhan 430030, China. ${ }^{6}$ Department of Radiation Oncology, UT Southwestern Medical Center, Dallas, TX 75390, USA. ${ }^{7}$ School of Biological Science and Technology, University of Jinan, Jinan, China.
\end{abstract}

\section{Received: 16 February 2017 Accepted: 7 August 2017}

\section{Published online: 22 August 2017}

\section{References}

1. Chen W, Zheng R, Baade PD, Zhang S, Zeng H, Bray F, Jemal A, Yu XQ, He J. Cancer statistics in China, 2015. CA Cancer J Clin. 2016;66:115-32.

2. Nakamura $\mathrm{H}$, Saji $\mathrm{H}$. Worldwide trend of increasing primary adenocarcinoma of the lung. Surg Today. 2014;44:1004-12.

3. Siegel RL, Miller KD, Jemal A. Cancer statistics, 2015. CA Cancer J Clin. 2015; 65:5-29.

4. Ambros V. The functions of animal microRNAs. Nature. 2004:431:350-5.

5. Calin GA, Croce CM. MicroRNA signatures in human cancers. Nat Rev Cancer. 2006:6:857-66.

6. Hayashita Y, Osada H, Tatematsu Y, Yamada H, Yanagisawa K, Tomida S, Yatabe Y, Kawahara K, Sekido Y, Takahashi T. A polycistronic microRNA cluster, miR-17-92, is overexpressed in human lung cancers and enhances cell proliferation. Cancer Res. 2005;65:9628-32.

7. Hatley ME, Patrick DM, Garcia MR, Richardson JA, Bassel-Duby R, van Rooij E, Olson EN. Modulation of K-Ras-dependent lung tumorigenesis by MicroRNA-21. Cancer Cell. 2010;18:282-93.

8. Garofalo M, Di Leva G, Romano G, Nuovo G, Suh SS, Ngankeu A, Taccioli C Pichiorri F, Alder H, Secchiero P, et al. miR-221\&222 regulate TRAll resistance and enhance tumorigenicity through PTEN and TIMP3 downregulation. Cancer Cell. 2009:16:498-509.

9. Takamizawa J, Konishi H, Yanagisawa K, Tomida S, Osada H, Endoh H, Harano T, Yatabe $Y$, Nagino M, Nimura $Y$, et al. Reduced expression of the let-7 microRNAs in human lung cancers in association with shortened postoperative survival. Cancer Res. 2004;64:3753-6.

10. Yang J, Lan H, Huang X, Liu B, Tong Y. MicroRNA-126 inhibits tumor cell growth and its expression level correlates with poor survival in non-small cell lung cancer patients. PLoS One. 2012;7:e42978.

11. Ke Y, Zhao W, Xiong J, Cao R. Downregulation of miR-16 promotes growth and motility by targeting HDGF in non-small cell lung cancer cells. FEBS Lett. 2013;587:3153-7.

12. Fernandez S, Risolino M, Mandia N, Talotta F, Soini Y, Incoronato M, Condorelli G, Banfi S, Verde P. miR-340 inhibits tumor cell proliferation and induces apoptosis by targeting multiple negative regulators of p27 in nonsmall cell lung cancer. Oncogene. 2015:34:3240-50.

13. Chen Z, Zeng H, Guo Y, Liu P, Pan H, Deng A, Hu J. miRNA-145 inhibits non-small cell lung cancer cell proliferation by targeting c-Myc. J Exp Clin Cancer Res. 2010;29:151.

14. Seol HS, Akiyama Y, Shimada S, Lee HJ, Kim TI, Chun SM, Singh SR, Jang SJ. Epigenetic silencing of microRNA-373 to epithelial-mesenchymal transition in non-small cell lung cancer through IRAK2 and LAMP1 axes. Cancer Lett. 2014;353:232-41.

15. Griffiths-Jones S, Grocock RJ, van Dongen S, Bateman A, Enright AJ. miRBase: microRNA sequences, targets and gene nomenclature. Nucleic Acids Res. 2006;34:D140-4.

16. Martinez I, Gardiner AS, Board KF, Monzon FA, Edwards RP, Khan SA. Human papillomavirus type 16 reduces the expression of microRNA-218 in cervical carcinoma cells. Oncogene. 2008;27:2575-82.

17. Davidson MR, Larsen JE, Yang IA, Hayward NK, Clarke BE, Duhig EE, Passmore LH, Bowman RV, Fong KM. MicroRNA-218 is deleted and downregulated in lung squamous cell carcinoma. PLoS One. 2010;5:e12560.

18. Tatarano S, Chiyomaru T, Kawakami K, Enokida H, Yoshino H, Hidaka H, Yamasaki T, Kawahara K, Nishiyama K, Seki N, Nakagawa M. miR-218 on the genomic loss region of chromosome 4p15.31 functions as a tumor suppressor in bladder cancer. Int J Oncol. 2011;39:13-21.

19. Xia H, Yan Y, Hu M, Wang Y, Wang Y, Dai Y, Chen J, Di G, Chen X, Jiang X. MiR-218 sensitizes glioma cells to apoptosis and inhibits tumorigenicity by regulating ECOP-mediated suppression of NF-kappaB activity. NeuroOncology. 2013;15:413-22.

20. Tie J, Pan Y, Zhao L, Wu K, Liu J, Sun S, Guo X, Wang B, Gang Y, Zhang Y, et al. MiR-218 inhibits invasion and metastasis of gastric cancer by targeting the Robo1 receptor. PLoS Genet. 2010;6:e1000879.

21. Lewis BP, Burge CB, Bartel DP. Conserved seed pairing, often flanked by adenosines, indicates that thousands of human genes are microRNA targets. Cell. 2005;120:15-20.

22. Wong $N$, Wang $X$. miRDB: an online resource for microRNA target prediction and functional annotations. Nucleic Acids Res. 2015;43:D146-52.

23. Krek A, Grun D, Poy MN, Wolf R, Rosenberg L, Epstein EJ, MacMenamin P, da Piedade I, Gunsalus KC, Stoffel M, Rajewsky N. Combinatorial microRNA target predictions. Nat Genet. 2005;37:495-500.

24. Kertesz M, lovino N, Unnerstall U, Gaul U, Segal E. The role of site accessibility in microRNA target recognition. Nat Genet. 2007;39:1278-84.

25. Eirew P, Kannan N, Knapp DJ, Vaillant F, Emerman JT, Lindeman GJ, Visvader $\mathrm{JE}$, Eaves CJ. Aldehyde dehydrogenase activity is a biomarker of primitive normal human mammary luminal cells. Stem Cells. 2012;30:344-8.

26. Huang CP, Tsai MF, Chang TH, Tang WC, Chen SY, Lai HH, Lin TY, Yang JC, Yang PC, Shih JY, Lin SB. ALDH-positive lung cancer stem cells confer resistance to epidermal growth factor receptor tyrosine kinase inhibitors. Cancer Lett. 2013;328:144-51.

27. Li JH, Liu S, Zhou H, Qu LH, Yang JH. starBase v2.0: decoding miRNA-ceRNA, miRNA-ncRNA and protein-RNA interaction networks from large-scale CLIPSeq data. Nucleic Acids Res. 2014;42:D92-7.

28. Yang JH, Li JH, Shao P, Zhou H, Chen YQ, Qu LH. starBase: a database for exploring microRNA-mRNA interaction maps from Argonaute CLIP-Seq and Degradome-Seq data. Nucleic Acids Res. 2011;39:D202-9.

29. Liu A, Liu Y, Li PK, Li C, Lin J. LLL12 inhibits endogenous and exogenous interleukin-6-induced STAT3 phosphorylation in human pancreatic cancer cells. Anticancer Res. 2011;31:2029-35.

30. Lin L, Hutzen B, Li PK, Ball S, Zuo M, DeAngelis S, Foust E, Sobo M, Friedman L, Bhasin D, et al. A novel small molecule, LLL12, inhibits STAT3 phosphorylation and activities and exhibits potent growth-suppressive activity in human cancer cells. Neoplasia. 2010;12:39-50.

31. Zhu K, Ding H, Wang W, Liao Z, Fu Z, Hong Y, Zhou Y, Zhang CY, Chen X. Tumor-suppressive miR-218-5p inhibits cancer cell proliferation and migration via EGFR in non-small cell lung cancer. Oncotarget. 2016;7:28075-85.

32. Szasz AM, Lanczky A, Nagy A, Forster S, Hark K, Green JE, Boussioutas A, Busuttil R, Szabo A, Gyorffy B. Cross-validation of survival associated biomarkers in gastric cancer using transcriptomic data of 1,065 patients. Oncotarget. 2016;7:49322-33.

33. Jansson MD, Lund AH. MicroRNA and cancer. Mol Oncol. 2012;6:590-610.

34. Di Leva G, Garofalo M, Croce CM. MicroRNAs in cancer. Annu Rev Pathol. 2014;9:287-314

35. Hayes J, Peruzzi PP, Lawler S. MicroRNAs in cancer: biomarkers, functions and therapy. Trends Mol Med. 2014;20:460-9. 
36. Torre LA, Bray F, Siegel RL, Ferlay J, Lortet-Tieulent J, Jemal A. Global cancer statistics, 2012. CA Cancer J Clin. 2015;65:87-108.

37. Thress KS, Brant R, Carr TH, Dearden S, Jenkins S, Brown H, Hammett T, Cantarini M, Barrett JC. EGFR mutation detection in ctDNA from NSCLC patient plasma: a cross-platform comparison of leading technologies to support the clinical development of AZD9291. Lung Cancer. 2015;90:509-15.

38. Du L, Schageman JJ, Irnov GL, Hammond SM, Minna JD, Gazdar AF, Pertsemlidis A. MicroRNA expression distinguishes SCLC from NSCLC lung tumor cells and suggests a possible pathological relationship between SCLCs and NSCLCs. J Exp Clin Cancer Res. 2010;29:75.

39. Matsubara H, Takeuchi T, Nishikawa E, Yanagisawa K, Hayashita Y, Ebi H, Yamada H, Suzuki M, Nagino M, Nimura Y, et al. Apoptosis induction by antisense oligonucleotides against miR-17-5p and miR-20a in lung cancers overexpressing miR-17-92. Oncogene. 2007;26:6099-105.

40. Liu X, Sempere LF, Ouyang H, Memoli VA, Andrew AS, Luo Y, Demidenko E, Korc M, Shi W, Preis M, et al. MicroRNA-31 functions as an oncogenic microRNA in mouse and human lung cancer cells by repressing specific tumor suppressors. J Clin Invest. 2010;120:1298-309.

41. Cui R, Meng W, Sun HL, Kim T, Ye Z, Fassan M, Jeon YJ, Li B, Vicentini C, Peng $Y$, et al. MicroRNA-224 promotes tumor progression in nonsmall cell lung cancer. Proc Natl Acad Sci U S A. 2015;112:E4288-97.

42. Kumar MS, Erkeland SJ, Pester RE, Chen CY, Ebert MS, Sharp PA, Jacks T. Suppression of non-small cell lung tumor development by the let-7 microRNA family. Proc Natl Acad Sci U S A. 2008:105:3903-8.

43. Kasinski AL, Slack FJ. miRNA-34 prevents cancer initiation and progression in a therapeutically resistant $\mathrm{K}$-ras and $\mathrm{p} 53$-induced mouse model of lung adenocarcinoma. Cancer Res. 2012;72:5576-87.

44. Miko E, Margitai Z, Czimmerer Z, Varkonyi I, Dezso B, Lanyi A, Bacso Z, Scholtz B. miR-126 inhibits proliferation of small cell lung cancer cells by targeting SLC7A5. FEBS Lett. 2011;585:1191-6.

45. Dutta P, Sabri N, Li J, Li WX. Role of STAT3 in lung cancer. JAKSTAT. 2014;3: e999503.

46. Lim CP, Cao X. Structure, function, and regulation of STAT proteins. Mol BioSyst. 2006;2:536-50

47. Alvarez JV, Greulich H, Sellers WR, Meyerson M, Frank DA. Signal transducer and activator of transcription 3 is required for the oncogenic effects of nonsmall-cell lung cancer-associated mutations of the epidermal growth factor receptor. Cancer Res. 2006;66:3162-8.

48. Deshmukh A, Deshpande K, Arfuso F, Newsholme P. Dharmarajan A. Cancer stem cell metabolism: a potential target for cancer therapy. Mol Cancer. 2016;15:69.

49. Takahashi RU, Miyazaki H, Ochiya T. The role of microRNAs in the regulation of cancer stem cells. Front Genet. 2014;4:295.

50. Hsu HS, Lin JH, Huang WC, Hsu TW, Su K, Chiou SH, Tsai YT, Hung SC. Chemoresistance of lung cancer stemlike cells depends on activation of Hsp27. Cancer. 2011;117:1516-28.

51. Li L, Zhao G. Downregulation of microRNA-218 relieves neuropathic pain by regulating suppressor of cytokine signaling 3. Int J Mol Med. 2016;37:851-8.

52. Zhang $\mathrm{S}$, Wang W, Wang E, Qiu X. SOCS3 expression is inversely correlated with Pyk2 in non-small cell lung cancer and exogenous SOCS3 inhibits proliferation and invasion of A549 cells. Pathology. 2012;44:434-40.

53. Lin YC, Lin CK, Tsai YH, Weng HH, Li YC, You L, Chen JK, Jablons DM, Yang

$C T$. Adenovirus-mediated SOCS3 gene transfer inhibits the growth and enhances the radiosensitivity of human non-small cell lung cancer cells. Oncol Rep. 2010;24:1605-12.

\section{Submit your next manuscript to BioMed Central and we will help you at every step:}

- We accept pre-submission inquiries

- Our selector tool helps you to find the most relevant journal

- We provide round the clock customer support

- Convenient online submission

- Thorough peer review

- Inclusion in PubMed and all major indexing services

- Maximum visibility for your research

Submit your manuscript at www.biomedcentral.com/submit
Biomed Central 